\title{
Immune Thrombocytopenia (ITP) in a Patient with Relapsing-Remitting Multiple Sclerosis (RRMS) Associated with b-Interferon-1 $\alpha$ Treatment
}

\author{
Vahid Shaygannejad ${ }^{1,2}$, Emad Fayyazi ${ }^{1,3}$, Omid Mirmosayyeb ${ }^{1,3}$, Nafiseh Toghianifar ${ }^{1,4^{*}}$ \\ ${ }^{1}$ Isfahan Neuroscience Research Center, Isfahan University of Medical Sciences, Isfahan, IRAN. \\ 2Department of Neurology, Isfahan University of Medical Sciences, Isfahan, IRAN. \\ ${ }^{3}$ Medical students' research Committee, Isfahan University of Medical Sciences, Isfahan, IRAN. \\ ${ }^{4}$ Non-communicable Disease Unit, Vice Chancellor for Clinical Affairs, Isfahan University of Medical Sciences, Isfahan, IRAN.
}

\section{BACKGROUND}

Immune thrombocytopenia is an isolated thrombocytopenia not related to other etiologies of thrombocytopenia. ${ }^{1}$ The diagnosis relies mainly on exclusion of other conditions. ${ }^{2,3}$ The incidence of ITP among adults has been estimated to be 3.3 per 100,000 persons in Europe according to a review study in $2009 .{ }^{4}$ ITP may be induced by drugs. ${ }^{2}$ In this type of ITP, a drug induces production of antibodies against platelets and causes destruction and reduction of platelets. ${ }^{2}$ This is usually transient and improves after withdrawal of the offending drug. ${ }^{1,2}$

Cinnovex is a b-interferon- $1 \alpha$ widely used in RRMS patients in Iran. Cinnovex is a biosimilar form of Avonex. ${ }^{5}$ Various studies have shown its efficacy in RRMS patients in Iran. ${ }^{5,6}$ The frequency of thrombocytopenia ranges from $0.1 \%$ to $8 \%$ in patients receiving b-interferon- $1 \alpha$ (http://www.cinnagen.com/ Product.aspx? $\mathrm{t}=2 \& \mathrm{l}=1 \& \mathrm{Id}=45 \& \mathrm{f}=2 \# \mathrm{ti}$ tle32). Thrombocytopenia has also been reported in patients receiving Cinnovex in Iran (Product Information. Avonex (interferon beta-1a)." Biogen, Cambridge,MA.) This paper reports a case of severe immune thrombocytopenia (ITP) in a RRMS patient receiving b-interferon- $1 \alpha$ (Cinnovex).

\section{CASE REPORT}

A 22 year old woman with a one-year history of RRMS referred to MS Clinic of Kashani Hospital, Isfahan, due to purpuric rash. She had developed the rash from 3 days before admission, first noticed periorally, and then spreading on her face and limbs, and rapidly spreading all over her body.

On Aug 2016 the patient developed blurred vision and was diagnosed as an attack of optic neuritis. Typical demyelinating plaques on brain and cervical cord MRI were noted. On Sept 2016, she was diagnosed with MS according to Mc Donald criteria supported with MRI findings. She received methyl prednisolone pulse therapy for 3 days. She was started on b-interferon- $1 \alpha$, IM $30 \mu \mathrm{g}$ weekly (Cinnovex, CinnaGen Co, Tehran, Iran). Weekly injections continued until she developed purpuric rash on March 2017. It was discontinued then. Previous counts of platelets were in the normal range, i.e. $285,000 / \mu \mathrm{L}$ on Dec 2016. At that time she was not receiving other medication except for oral vitamin D pearls 50,000 IU weekly. The symptoms occurred two days after the last injection of Cinnovex.

On examination, there was a purpuric rash, varying in size from $2 \mathrm{~mm}$ to $5 \mathrm{~mm}$, extending all over her body including wet perioral petechiae. There was no other abnormality in the physical examination, such as splenomegaly, hepatomegaly or lypmhadenopathy. The neurologic exam was normal. The results of blood samples were as follows: white

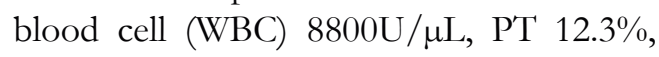
PTT 35Sec, INR 1, Hemoglobin 13.9g/dL, platelet $6000 / \mu \mathrm{L}$, TSH $0.22 \mu \mathrm{lU} / \mathrm{mL}$. Blood
Submission Date: 29-09-2017; Revision Date: 08-11-2017; Accepted Date: 15-12-2017

DOI: 10.5530/ijper.52.3.62

Correspondence: Nafiseh Toghianifar, MD, Neurologist

Isfahan Neuroscience Research Center, Soffeh St, Isfahan, IRAN.

Phone: +989132048818

E-mail: n.toghiani@gmail.com

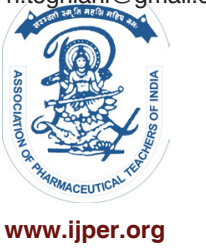


tests were negative for HBs-Ag, HCV-Ab, ANA, ANCA, anticardiolipin(acL), Lupus anticoagulant (La) and antiphospholipid antibodis. Anti-thrombocyte antibodies are not available to measure in Iran and ITP diagnosis is based on clinical and other laboratory criteria.

A diagnosis of immune thrombocytopenia (ITP) was made. Cinnovex was stopped. She received intravenous methylprednisolone $500 \mathrm{mg}$, for 2 days, and intravenous immungloboulin (IVIG), 55 gm once. While the platelet count was $6,000 / \mu \mathrm{L}$ at admission, and dropped to $4,000 / \mu \mathrm{L}$ on second day, it increased $72,000 / \mu \mathrm{L}$ on the 4th day of treatment. On 2-week follow-up it increased to $318,000 / \mu \mathrm{L}$. She was started on CinnoMer (Glatiramer Acetate, CinnaGen Co.). There was no recurrence of thrombocytopenia in the following follow-up visits.

\section{DISCUSSION}

This is one the first reports of ITP after receiving Cinnovex. Thrombocytopenia has been reported in patients receiving b-interferon- $1 \alpha$ including Cinnovex. In some cases, platelet counts were below $10,000 / \mu \mathrm{L}$. Some cases recurred with restarting medication. Our patient had severe ITP which is not a common side effect of this medication among RRMS patients.

A case-control study on 187 MS patients showed a higher prevalence of thrombocytopenia in RRMS patients but not in other MS subtypes. It was associated with lower Expanded Disability Status Scale (EDSS) and shorter duration of disease. ${ }^{7}$ Also, it was higher in patients receiving disease modifying treatments including b-interferon. Our patient had low EDSS and 1-year history of RRMS. She was receiving Iranian brand of b-interferon- $1 \alpha$. The exact mechanism of thrombocytopenia in patients receiving b-interferon is not clear. While ITP is considered an immune-mediated decrease in platelet counts, its cellular and molecular mechanism has not been studied specifically in patients receiving b-interferon. ${ }^{3,4}$

ITP is more common among women especially in adulthood. Similarly, our patient was a 22 year old woman. A large study by Segal et al on 1169 ITP patients showed MS is 25 times more prevalent in childhood ITP cases. ${ }^{3}$ As they reported, the patients in their study were not receiving b-interferon- $1 \alpha$. However, Segal et al doubted the accuracy of this report and related it to incompleteness of pharmacy reports. ${ }^{3}$ A prospective study may be helpful in clarifying the relation between ITP and MS, as platelets are involved in immune mechanisms and inflammation. (Wachowicz B, Morel A, Miller E, Saluk J. The physiology of blood platelets and changes of their biological activities in multiple sclerosis. Acta Neurobiol Exp (Wars). 2016;76(4):269-81.)

\section{CONCLUSION}

ITP must be considered in RRMS patients, especially those receiving b-interferon- $1 \alpha$ that refer with hematologic signs or symptoms indicating platelet disorders. Patients should be monitored periodically for platelet counts as recommended. Further studies are needed to investigate exact underlying mechanism of ITP in patients receiving Cinnovex.

\section{ACKNOWLEDGEMENT}

The authors wish to thank the patient for her cooperation and permission to publish related data.

\section{CONFLICT OF INTEREST}

On behalf of all authors, the corresponding author declares there is no conflict of interest. This study was carried out without any financial support from private or public institutions.

\section{ABBREVIATIONS}

ITP: Immune Thrombocytopenia; RRMS: Relapsing Remitting Multiple Sclerosis; WBC: White Blood Cell, aCL: anti-Cardiolipin; La: Lupus anticoagulant; IVIG: Intravenous Immuneglobulin; EDSS: Expanded Disability Status Scale.

\section{REFERENCES}

1. Michel M. Immune thrombocytopenic purpura: epidemiology and implications for patients. European journal of haematology Supplementum. 2009;82(s71):3-7.

2. Rodeghiero F, Stasi R, Gernsheimer T, Michel M, Provan D, Arnold DM, et al. Standardization of terminology, definitions and outcome criteria in immune thrombocytopenic purpura of adults and children: report from an international working group. Blood. 2009;113(11):2386-93.

3. Segal JB, Powe NR. Prevalence of immune thrombocytopenia: analyses of administrative data. Journal of thrombosis and haemostasis: JTH. 2006;4(11):2377-83.

4. Terrell DR, Beebe LA, Vesely SK, Neas BR, Segal JB, George JN. The incidence of immune thrombocytopenic purpura in children and adults: A critical review of published reports. American journal of hematology. 2010;85(3):174-80.

5. Abolfazli R, Hosseini A, Gholami K, Javadi MR, Torkamandi H, Emami S. Quality of Life Assessment in Patients with Multiple Sclerosis Receiving Interferon Beta-1a: A Comparative Longitudinal Study of Avonex and Its Biosimilar CinnoVex. ISRN Neurol. 2012;2012:786526.

6. Shahkarami MA, Vaziri B, Salami S, Harandi AA, Oger J. Neutralizing antibodies in multiple sclerosis patients on weekly intramuscular Avonex and biosimilar interferon beta-1a (CinnoVex): comparing results of measurements in two different laboratories. J Immunol Methods. 2013;388(1-2):46-8.

7. Koudriavtseva T, Plantone D, Renna R, Mandoj C, Giannarelli D, Mainero C. Interferon-beta therapy and risk of thrombocytopenia in multiple sclerosis patients. Neurol Sci. 2015;36(12):2263-8. 


\section{PICTORIAL ABSTRACT}

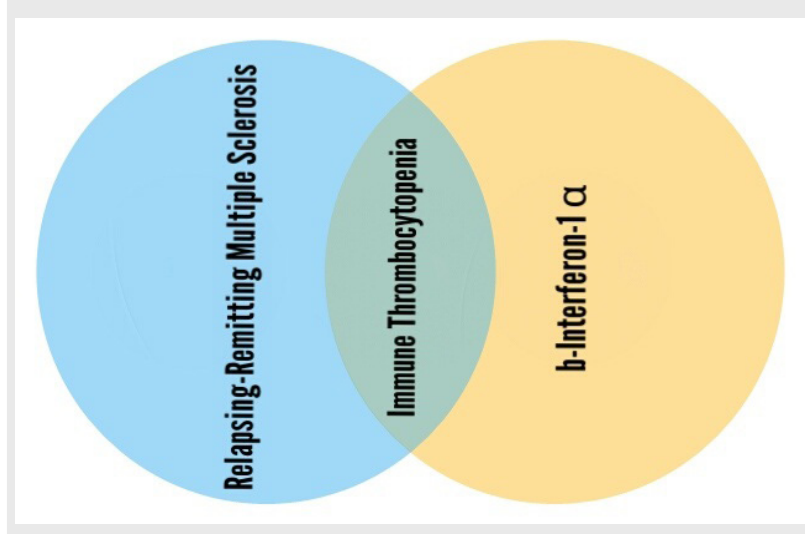

\section{SUMMARY}

This paper reports a case of severe immunethrombocytopenia (ITP) associated with b-interferon- $1 \alpha$ (Cinnovex) in a patient with relapsing remitting multiples clerosis (RRMS). A 22 year old woman with a one-year history of RRMS referred to MS Clinic due to purpuricrash. The platelet count was $6,000 / \mu \mathrm{L}$ at admission. A diagnosis ITP was made. Cinnovex was stopped. She received intravenous methylprednisolone $500 \mathrm{mg} / \mathrm{d}$, for2days, and intravenous immungloboulin (IVIG), 55mg.Thrombocytopeniare solved in the following days. She was started on CinnoMer (GlatiramerAcetate, CinnaGenCo.) for MSmanagement. There was no recurrence of thrombocytopenia in the next follow-up visits.

Cite this article: Shaygannejad V, Fayyazi E, Mirmosayyeb O, Immune Thrombocytopenia (ITP) in a Patient with Relapsing-Remitting Multiple Sclerosis (RRMS) Associated with b-Interferon- $1 \alpha$ Treatment. Indian J of Pharmaceutical Education and Research. 2018;52(3):540-2. 